\title{
Motion Models for use with the Maneuvering Ballistic Missile Tracking Estimators
}

\author{
Jinwhan Kim ${ }^{*}$ and P. K. Menon ${ }^{\dagger}$ \\ Optimal Synthesis Inc., Los Altos, CA 94022 \\ and \\ Ernest Ohlmeyer* \\ Aero Science Applications, King George, VA 22485
}

\begin{abstract}
Ballistic target interception requires high accuracy in order to achieve kinetic hit-to-kill performance. Since the task becomes more challenging when the target performs complex maneuvers, highly accurate state estimators are indispensable for successful ballistic target interception. This research focuses on developing filter motion models which can reproduce a wide variety of target motions which include intelligent evasive maneuvers. The proposed filtering technique is expected to improve tracking performance for a maneuvering target undergoing unpredictable motions, which will eventually reduce the miss distance of the ballistic target interception. The feasibility of the developed motion models is demonstrated through numerical tracking simulations, and the performance is compared with that of conventional filter motion models.
\end{abstract}

\section{Introduction}

$\mathrm{T}$

HE evolving nature of ballistic missile threats drives the need for sophisticated interceptor flight control systems. Successful interception requires three key subsystem components: estimation, guidance, and control. Among these, target state estimation performance is critically important to the overall interception, since it determines the interception performance bound. That is, no matter how sophisticated the flight control system is, the statistical miss distance error cannot be reduced below the performance guaranteed by the estimator ${ }^{1}$.

The target state estimation is a nonlinear estimation problem due to the nature of system dynamics and the engagement geometry. Even when the sensor noise is Gaussian, the nonlinear dynamics can create non-Gaussian behavior in the estimator ${ }^{2}$. Online recursive filtering techniques which were originally designed for linear/Gaussian systems have commonly been applied to the nonlinear target state estimation problems through linearization of the system dynamics. Such an approximation scheme often works well for estimating the states of non-maneuvering targets $^{3,4}$. However, these filtering techniques may not show consistent and reliable performance for estimating the states of a target undergoing complex or unpredictable maneuvers. This is an important issue in ballistic missile interception which requires highly accurate estimator performance to achieve the small miss distances required for kinetic kill.

In particular, target modeling is an extremely important part of the tracking filter design. Using good target motion models is often much more effective and advantageous in improving the overall tracking filter performance than employing more sophisticated filter algorithms or sensor models. A body of literature on modeling motion models for a maneuvering target is available. Among them, the so-called wide-band models are commonly used for tracking a maneuvering target, which can cover a large set of target motion. However, they are crude and are known to be unsuitable for an accurate estimation of maneuvering ballistic target states. In fact, if any prior information on the target such as missile type and dynamic characteristics are available, they can effectively be used to better represent the target model at its maneuvers. Full exploitation of such information will improve the overall state estimation performance.

\footnotetext{
* Presently Assistant Professor, KAIST, Daejon, Korea, Senior Member AIAA.

${ }^{\dagger}$ Chief Scientist and President, 95 First Street, Fellow AIAA.

* Principal Scientist, Associate Fellow AIAA.
} 
In this study, research effort will specifically be focused on ballistic target modeling. Ballistic targets possess quite distinctive motion properties compared to other maneuvering targets. A 6-DOF dynamic model of a ballistic target is derived and its motion characteristics are analyzed. Such knowledge is used to set up an appropriate target dynamics model for achieving improved tracking performance.

Section II describes the related work done previously. Section III presents the mathematical model of a ballistic target based on aerodynamic analysis, and Section IV describes the target motion models for formulating online recursive filters. The performance of the motion models are evaluated and compared in Section 0, and the conclusions from the present research are given in Section VI.

\section{Related Work}

A significant amount of research has been carried out over the past four decades on estimating the states of maneuvering targets ${ }^{5-8}$. Particularly, comprehensive surveys on a number of target models for modeling a maneuvering target are available in References 7 and 8. Even without target-specific information, a large set of target motions can be covered by wide-band models such as the acceleration model, the jerk model and the Singer model ${ }^{7}$. These wide-band models are known to be effective for non-maneuvering or slowly maneuvering targets. However, they are known to be unsuitable for the accurate estimation of a ballistic target which may perform various types of intended or unintended evasive maneuvers.

To capture such unpredictable evasive motions, a lot of research has been devoted to multiple model approaches such as the Generalized Pseudo-Bayesian (GPB) and the Interacting Multiple Model (IMM) technique ${ }^{6,9-11}$. The basic assumptions of the multiple model algorithms are that the system dynamics can be represented as one of a finite number of modes, and mode switching (or jumping) may take place between the pre-defined target dynamics models. Though mode switching is a nonlinear behavior, the filter for an individual model is often based on a classical filtering algorithm such as EKF or UKF which may still have fundamental limitations in its application to nonlinear/non-Gaussian systems. However, a more fundamental limitation of the multiple model method is that it assumes the system to be in one of the built-in modes of the system. Consequently, the filter displays resistance to changes in target maneuvers, leading to large estimation errors.

Another approach that may be useful is the Finite Impulse Response (FIR) target model-based filtering, ${ }^{3,12}$. It has been widely used in the area of signal processing and system identification including speech recognition/prediction, adaptive noise cancellation, and behavior prediction of dynamic systems. The technique can also be applied to the online motion prediction of targets ${ }^{13}$. The order of the FIR filter is required to be specified in advance, which is a design parameter. Consequently, the higher the order of the FIR filter, the richer is the set of target motion that can be represented. While the conventional linear FIR filter approach for target modeling is highly flexible, a large number of terms are required to represent complex target behavior, and this flexibility comes at the price of the risk of overfitting, which may lead to the lack of state observability ${ }^{3,6}$.

More recently, the motion model for the state-parameter estimation of a spiraling target was proposed ${ }^{5}$ and its tracking performance with the use of several nonlinear filtering techniques was evaluated and compared ${ }^{14}$. These studies were performed based on the observation that some tactical ballistic missiles exhibit violent spiraling motion during reentry to the atmosphere.

\section{Mathematical Modeling of a Ballistic Target}

Dynamic modeling of the target is an essential part of state estimation system design. In this section, a 6-DOF nonlinear aerodynamic model for a reentering ballistic target is described. The focus is on modeling the spiraling motions sometimes observed for these targets. This motion is induced by the aerodynamic instability arising from roll-resonance ${ }^{15-18}$.

The ultimate requirement for the target motion model is that it should be simple enough to be computationally efficient while being flexible enough to allow for a wide range of expected target motions. Based on the dynamic analysis in this section, target motion models for estimator design are formulated in the next section.

\section{A. Equations of Motion}

The equations of motion for 6-DOF rigid body dynamics in the body-fixed frame ${ }^{19}$ provide a basic framework to represent the generalized system dynamics of a ballistic target.

Force equilibrium along the body axes yield: 


$$
\left[\begin{array}{l}
X \\
Y \\
Z
\end{array}\right]=\frac{d}{d t}(m \vec{V})=m(\dot{\vec{V}}+\vec{\omega} \times \vec{V})=m\left[\begin{array}{c}
\dot{u}+q w-r v \\
\dot{v}-p w+r u \\
\dot{w}+p v-q u
\end{array}\right]
$$

where $X, Y$ and $Z$ are the external forces acting on the ballistic target, and $m$ is the target mass. The variables $u, v$ and $w$ are the velocities and $p, q$ and $r$ are the angular velocities in body-fixed coordinates. Moment equilibrium about the body axes produces:

$$
\left[\begin{array}{c}
L \\
M \\
N
\end{array}\right]=\frac{d}{d t}(\vec{H})=\bar{I} \dot{\vec{\omega}}+\vec{\omega} \times(\bar{I} \vec{\omega})=\left[\begin{array}{c}
I_{x} \dot{p} \\
I_{y} \dot{q}+p r\left(I_{x}-I_{z}\right) \\
I_{z} \dot{r}+p q\left(I_{y}-I_{x}\right)
\end{array}\right]
$$

Here, the mass moment of inertia matrix is assumed to be about the principal axes (i.e., the matrix is diagonal), and $I_{y}=I_{z}$ for a body that is symmetric along the $x$-axis. Given the external forces and moments (i.e., $X, Y, Z, L, M$, $N$ ), the linear and angular velocities in body-fixed coordinates (i.e., $u, v, w, p, q, r$ ) can be calculated, and they can be integrated to yield the position and orientation in the earth-fixed frame (i.e., $x, y, z, \phi, \theta, \psi)$ through the Euler angle transformation.

The forces and moments acting on the target during reentry arise from the Earth's gravity and aerodynamics. They can be expressed as:

$$
\left[\begin{array}{c}
X \\
Y \\
Z \\
L \\
M \\
N
\end{array}\right]=\left[\begin{array}{c}
X_{G} \\
Y_{G} \\
Z_{G} \\
0 \\
0 \\
0
\end{array}\right]+\left[\begin{array}{c}
X_{u^{2}} u^{2}+X_{v} v+X_{w} w+X_{p} p+X_{q} q+X_{r} r \cdots \\
Y_{u} u+Y_{v} v+Y_{w} w+Y_{p} p+Y_{q} q+Y_{r} r \cdots \\
Z_{u} u+Z_{v} v+Z_{w} w+Z_{p} p+Z_{q} q+Z_{r} r \cdots \\
L_{u} u+L_{v} v+L_{w} w+L_{p} p+L_{q} q+L_{r} r \cdots \\
M_{u} u+M_{v} v+M_{w} w+M_{p} p+M_{q} q+M_{r} r \cdots \\
N_{u} u+N_{v} v+N_{w} w+N_{p} p+N_{q} q+N_{r} r \cdots
\end{array}\right]
$$

In Eqn. (3), the gravity force has three components since the formulation is in terms of the body-fixed frame. Also, a number of aerodynamic derivatives are used to represent the aerodynamic forces and moments ${ }^{20,21}$. These derivatives mainly depend on the assumed geometry of the target.

Considering the narrow interception time window, the limited number of measurements, and potential observability problems, it is extremely difficult to estimate all the aerodynamic derivatives. The problem becomes even more challenging when the target is maneuvering, since all the force and moment derivatives may now be time-varying.

\section{B. Roll-Resonance during Ballistic Reentry}

Although the system dynamics given in the previous subsection is too detailed for the filter design, it can be used as the basis for describing and predicting typical behaviors of the ballistic targets. It can also provide physical intuition for deriving an efficient motion model to be used in the estimator derivation. In particular, this model can be used to analytically investigate the roll-resonance phenomenon induced by aerodynamic instability during the ballistic target reentry ${ }^{15-18}$.

In order to focus on the spiraling behavior in the lateral plane perpendicular to the target's direction of motion, the roll rate is assumed to be in quasi-steady state. That is, $u$ and $p$ are non-zero, but are assumed to be constant over some small time interval. The roll motion with non-zero $p$ is assumed to have been induced by unintentional configurational asymmetries. Also, from among a number of aerodynamic force/moment derivatives, several dominant components related to the roll-resonance are selected and considered for the current analysis. The resulting equations of motion in the lateral plane are:

$$
\left[\begin{array}{c}
m\left(\dot{v}-p_{s} w+r u_{s}\right) \\
m\left(\dot{w}+p_{s} v-q u_{s}\right) \\
I \dot{q}+p_{s} r\left(I_{x}-I\right) \\
I \dot{r}+p_{s} q\left(I-I_{x}\right)
\end{array}\right]=\left[\begin{array}{c}
Y_{v} v \\
Z_{w} w \\
M_{0}+M_{w} w+M_{q} q \\
N_{0}+N_{v} v+N_{r} r
\end{array}\right]
$$

Here, $u_{s}$ and $p_{s}$ are the steady-state linear and angular velocities about the body-fixed longitudinal axis. $M_{0}$ and $N_{0}$ are the aerodynamic moments induced by configurational asymmetries in the pitch and yaw directions. 
From the axisymmetry of the target: $I_{y}=I_{z}=I$. This condition also gives:

$$
Y_{v}=Z_{w}, M_{w}=-N_{v}, M_{q}=N_{r}
$$

It is often advantageous and convenient to express the lateral velocity components $v$ and $w$ in terms of the angles of attack, $\beta$ and $\alpha$. They are related as

$$
\begin{aligned}
& v=u_{s} \sin \beta \approx u_{s} \beta \\
& w=u_{s} \sin \alpha \approx u_{s} \alpha
\end{aligned}
$$

and the aerodynamic coefficients are related as

$$
Y_{v} v=Y_{\beta} \beta, \quad Z_{w} w=Z_{\alpha} \alpha, M_{w} w=M_{\alpha} \alpha, \quad N_{v} v=N_{\beta} \beta
$$

Note that: $Y_{\beta}=Z_{\alpha}, M_{\alpha}=-N_{\beta}, M_{q}=N_{r}$ due to axisymmetry. Thus, Equation (4) can be rewritten as:

$$
\left[\begin{array}{c}
\dot{\beta}-p_{s} \alpha+r \\
\dot{\alpha}+p_{s} \beta-q \\
\dot{q}-p_{s} r(1-\mu) \\
\dot{r}+p_{s} q(1-\mu)
\end{array}\right]=\left[\begin{array}{c}
Z_{\alpha} \beta /\left(m u_{s}\right) \\
Z_{\alpha} \alpha /\left(m u_{s}\right) \\
\left(M_{0}+M_{\alpha} \alpha+M_{q} q\right) / I \\
\left(N_{0}-M_{\alpha} \beta+M_{q} r\right) / I
\end{array}\right]
$$

where $\mu=I_{x} / I$. The above equation can be reduced into two second-order ordinary differential equations as:

$$
\left[\begin{array}{ll}
1 & 0 \\
0 & 1
\end{array}\right]\left[\begin{array}{l}
\ddot{\alpha} \\
\ddot{\beta}
\end{array}\right]+\left[\begin{array}{cc}
b_{1} & b_{2} \\
-b_{2} & b_{1}
\end{array}\right]\left[\begin{array}{c}
\dot{\alpha} \\
\dot{\beta}
\end{array}\right]+\left[\begin{array}{cc}
k_{1} & k_{2} \\
-k_{2} & k_{1}
\end{array}\right]\left[\begin{array}{l}
\alpha \\
\beta
\end{array}\right]=\left[\begin{array}{c}
-N_{0} / I \\
M_{0} / I
\end{array}\right]
$$

where

$$
\begin{aligned}
& b_{1}=-\frac{M_{q}}{I}-\frac{Z_{\alpha}}{m u_{s}} \\
& b_{2}=p_{s}(2-\mu) \\
& k_{1}=-p_{s}^{2}(1-\mu)-\frac{M_{\alpha}}{I}+\frac{M_{q} Z_{\alpha}}{I m u_{s}} \\
& k_{2}=-p_{s}\left\{(1-\mu) \frac{Z_{\alpha}}{m u_{s}}+\frac{M_{q}}{I}\right\}
\end{aligned}
$$

Eqn. (9) can also be expressed in a simpler form using the complex-variable notation ${ }^{17}$ :

$$
\ddot{\delta}+\left(b_{1}+i b_{2}\right) \dot{\delta}+\left(k_{1}+i k_{2}\right) \delta=i M^{*} / I
$$

where

$$
\delta=\beta+i \alpha \quad M^{*}=M_{0}+i N_{0}
$$

The solution to the linear dynamic equation in Eqn. (10) has the form:

$$
\delta=C_{1} e^{\left(\lambda_{1}+i \omega_{1}\right) t}+C_{2} e^{\left(\lambda_{2}+i \omega_{2}\right) t}+C_{3}
$$

The steady-state solution term $C_{3}$ can be obtained from Eqn. (9) or Eqn. (10), which determines the steadysteady angle of attack called the rolling trim.

It is worthwhile examining the effects of various parameters in Eqn. (9). If the roll rate $p_{s}$ is close to zero and $N_{0}$ and $M_{0}$ are negligible, the pitch and yaw motions are decoupled. In this case, both $b_{1}$ and $k_{1}$ become positive which is the necessary and sufficient condition for the second order dynamic system to be stable. To be more specific, the aerodynamic derivatives, $M_{q}$ and $Z_{\alpha}$ are always negative, and thus the system damping term, $b_{1}$ is always positive. The parameter $k_{1}$ is also positive since $M_{\alpha}$ is generally a large negative number for stable vehicles. As $p_{s}$ increases, the system's stability is affected in a complex manner, and when the roll-induced circular motion hits the pitch/yaw natural frequency, the roll resonance occurs. In particular, the parameters $N_{0}$ and $M_{0}$, which vary depending on the roll angle and the angles of attack, play critical roles in the overall system stability.

\section{Target Motion Models}

The full 6-DOF nonlinear motion model for describing the complex behavior of a ballistic target during reentry was presented in the previous section. In this section, candidate motion models for the tracking filter are discussed. 
The central requirement for the filter motion model is that the model should be simple enough to be computationally efficient and flexible enough to allow for a wide range of expected target motions.

Several target models are available for tracking both maneuvering and non-maneuvering targets ${ }^{6,7}$. Some of the widely-used target models are white-noise acceleration models, Wiener-process acceleration models, Singer acceleration models, and jerk models. These models can be classified as the wide-band models which can capture a wide range of target motions. However, generalization comes at the price of sacrificing motion fidelity. Ballistic targets have several specific behavioral characteristics which can be exploited to derive more efficient and effective estimators ${ }^{8}$.

The target model of interest in the present research must be capable of generating maneuvers such as weaving, spiraling and random telegraph motions. Five target motion models which have the potential to serve as the basis for estimator design are described in the following subsections. In particular, based on the discussion in Section III, a new motion model, called the generalized snap model, is derived and presented here as the fifth target motion model in Subsection E.

\section{A. White Noise Acceleration (WNA) Model}

The WNA model is one of the most commonly used wide-band models for describing motions of physical systems ${ }^{6}$. The model assumes that forces acting on the system and the resulting accelerations can be represented as white-Gaussian processes. This model is commonly used for tracking non-maneuvering targets whose motions deviate little from the straight line path.

$$
\left[\begin{array}{c}
\dot{x} \\
\dot{y} \\
\dot{z} \\
\ddot{x} \\
\ddot{y} \\
\ddot{z}
\end{array}\right]=\left[\begin{array}{llllll}
0 & 0 & 0 & 1 & 0 & 0 \\
0 & 0 & 0 & 0 & 1 & 0 \\
0 & 0 & 0 & 0 & 0 & 1 \\
0 & 0 & 0 & 0 & 0 & 0 \\
0 & 0 & 0 & 0 & 0 & 0 \\
0 & 0 & 0 & 0 & 0 & 0
\end{array}\right]\left[\begin{array}{c}
x \\
y \\
z \\
\dot{x} \\
\dot{y} \\
\dot{z}
\end{array}\right]+\left[\begin{array}{c}
0 \\
0 \\
0 \\
n_{1} \\
n_{2} \\
n_{3}
\end{array}\right]
$$

Here, $n_{(\cdot)}$ is white Gaussian; $n \sim N\left(0, \sigma^{2}\right)$. As seen in Eqn. (12), the model has a simple and general structure. The WNA model can be a practical choice if the target dynamics are unknown, since the model can cover a wide range of motions. However, it does not allow the incorporation of any prior information about the target maneuvering characteristics into the filter structure, and often exhibits mediocre or disappointing performance for agile targets.

\section{B. Wiener Process Acceleration (WPA) Model}

Another well-known wide-band model is the Wiener process acceleration model ${ }^{6}$ which is also called the jerk model. This assumes that the time derivatives of accelerations or jerk can be represented as white-Gaussian noise.

$$
\left[\begin{array}{c}
\dot{x} \\
\dot{y} \\
\dot{z} \\
\ddot{x} \\
\ddot{y} \\
\ddot{z} \\
\dddot{x} \\
\dddot{y} \\
\dddot{z}
\end{array}\right]=\left[\begin{array}{lllllllll}
0 & 0 & 0 & 1 & 0 & 0 & 0 & 0 & 0 \\
0 & 0 & 0 & 0 & 1 & 0 & 0 & 0 & 0 \\
0 & 0 & 0 & 0 & 0 & 1 & 0 & 0 & 0 \\
0 & 0 & 0 & 0 & 0 & 0 & 1 & 0 & 0 \\
0 & 0 & 0 & 0 & 0 & 0 & 0 & 1 & 0 \\
0 & 0 & 0 & 0 & 0 & 0 & 0 & 0 & 1 \\
0 & 0 & 0 & 0 & 0 & 0 & 0 & 0 & 0 \\
0 & 0 & 0 & 0 & 0 & 0 & 0 & 0 & 0 \\
0 & 0 & 0 & 0 & 0 & 0 & 0 & 0 & 0
\end{array}\right]\left[\begin{array}{c}
x \\
y \\
z \\
\dot{x} \\
\dot{y} \\
\dot{z} \\
\ddot{x} \\
\ddot{y} \\
\ddot{z}
\end{array}\right]+\left[\begin{array}{c}
0 \\
0 \\
0 \\
0 \\
0 \\
0 \\
n_{1} \\
n_{2} \\
n_{3}
\end{array}\right]
$$

Compared with the WNA model, this model is better suited for representing the higher-bandwidth motion of a maneuvering target. However, it still does utilize any system-specific information about the target dynamics. Thus, estimation errors are unavoidable while tracking highly maneuvering targets.

\section{Ballistic Reentry Vehicle (BRV) Model}

The foregoing models are very general, and can be used for any type of targets since they assume no targetspecific information. As observed at the beginning of this section, this generality comes at the price of limited tracking performance and estimation accuracy. 
Since the forces acting on a ballistic reentry vehicle are composed of gravitational and aerodynamic forces, this information can be used to improve the performance of the WPA model. The acceleration terms of the ballistic reentry vehicle ${ }^{8}$ can be expressed as follows:

$$
\left[\begin{array}{c}
\ddot{x} \\
\ddot{y} \\
\ddot{z}
\end{array}\right]=\left[\begin{array}{l}
0 \\
0 \\
g
\end{array}\right]+Q T_{E / B}(\gamma, \chi)\left[\begin{array}{l}
a_{x} \\
a_{y} \\
a_{z}
\end{array}\right]
$$

where, $Q$ is the dynamic pressure that can be expressed by:

$$
Q=\frac{1}{2} \rho\left(\dot{x}^{2}+\dot{y}^{2}+\dot{z}^{2}\right)=\frac{1}{2} \rho V^{2}
$$

The gravitational acceleration $g$ and the density $\rho$ are functions of altitude. Three unknown parameters, $a_{x}, a_{y}$ and $a_{z}$ representing the acceleration components acting on the body in the non-rotating body-fixed coordinate system, are assumed to follow Wiener processes. The variable $a_{x}$ is associated with the axial acceleration component, inversely proportional to the ballistic coefficient. The variables $a_{y}$ and $a_{z}$ represent the two normal acceleration components.

Here, a non-rotating body-fixed coordinate system whose $x$-axis is tangential to the target trajectory is used for coordinate transformation. The coordinate transformation matrix $T_{E / B}$ is represented as a function of the flight path angle $\gamma$ and heading angle $\chi$ calculated using measured velocity vector components of the target as

$$
T_{E / B}=\left[\begin{array}{ccc}
\cos \gamma \cos \chi & -\sin \chi & \sin \gamma \cos \chi \\
\cos \gamma \sin \chi & \cos \chi & \sin \gamma \sin \chi \\
-\sin \gamma & 0 & \cos \gamma
\end{array}\right]
$$

where

$$
\begin{aligned}
& \gamma=\tan ^{-1}\left(-\frac{\dot{z}}{\sqrt{\dot{x}^{2}+\dot{y}^{2}}}\right) \\
& \chi=\tan ^{-1}\left(\frac{\dot{y}}{\dot{x}}\right)
\end{aligned}
$$

\section{Spiral Motion Model}

During the reentry to the atmosphere, certain ballistic missiles are known to undergo violent spiraling motions induced by aerodynamic instability in the yaw/pitch modes. The proposed spiral motion model can improve the tracking of a ballistic target undergoing spiraling motions.

Aerodynamic forces acting on the target can be divided into two major components: 1) drag acting along the direction of the velocity vector, and 2) normal force acting in a plane perpendicular to the velocity vector. Spiraling motion can be approximated by the normal acceleration vector rotating at a certain rate within the normal plane. The associated dynamic model is given by the following equations ${ }^{14}$ :

$$
\left[\begin{array}{c}
\ddot{x} \\
\ddot{y} \\
\ddot{z}
\end{array}\right]=\left[\begin{array}{l}
0 \\
0 \\
g
\end{array}\right]+Q T_{E / B}(\gamma, \chi)\left[\begin{array}{c}
-C_{D m} \\
C_{N m} \cos \varphi \\
C_{N m} \sin \varphi
\end{array}\right]=\left[\begin{array}{l}
0 \\
0 \\
g
\end{array}\right]+Q T_{E / B}(\gamma, \chi)\left[\begin{array}{c}
-C_{D m} \\
Z_{1} \\
Z_{2}
\end{array}\right]
$$

where

$$
\left[\begin{array}{l}
\dot{Z}_{1} \\
\dot{Z}_{2}
\end{array}\right]=\left[\begin{array}{cc}
0 & -\omega \\
\omega & 0
\end{array}\right]\left[\begin{array}{l}
Z_{1} \\
Z_{2}
\end{array}\right]
$$

Here, $Z_{1}$ and $Z_{2}$ are harmonic oscillator terms. The drag parameter $C_{D m}$ and the spiraling frequency, $\omega$ are assumed to follow Wiener processes.

\section{E. Generalized Snap Model}

This model is motivated by the roll resonance analysis results described in Section B. The underlying motion model structure is based on the BRV model in C. 


$$
\left[\begin{array}{c}
\ddot{x} \\
\ddot{y} \\
\ddot{z}
\end{array}\right]=\left[\begin{array}{l}
0 \\
0 \\
g
\end{array}\right]+Q T_{E / B}(\gamma, \chi)\left[\begin{array}{l}
a_{x} \\
a_{y} \\
a_{z}
\end{array}\right]
$$

Here, $Q$ is the dynamic pressure and $T_{E / B}$ is the coordinate transformation matrix, which are defined in Eqs. (15) and (16), respectively.

In this model, the axial (or longitudinal) acceleration is still assumed to follow a Wiener process. However, the second derivatives of the accelerations, which are the fourth derivatives of the position vector, called snap or jounce, in the lateral directions are expressed using a pair of second order ordinary differential equations. This representation was inspired by the central mechanism of spiraling motion shown in Eqn. (9). The proposed motion model, termed as the generalized snap (GS) model, is expected to effectively capture various types of evasive motion including random telegraph and oscillatory motions such as spiraling and weaving.

$$
\begin{aligned}
& \dot{a}_{x}=n_{x} \\
& \ddot{a}_{y}=b_{y} \dot{a}_{y}+k_{y} a_{y}+n_{y} \\
& \ddot{a}_{z}=b_{z} \dot{a}_{z}+k_{z} a_{z}+n_{z}
\end{aligned}
$$

Here, the coupling between the lateral acceleration components is not considered since it would make the motion model overly complex and potentially unobservable.

\section{F. Summary of the Candidate Target Motion Models}

The five target motion models discussed in the foregoing sections are summarized in Table 1. The performance comparisons between tracking filters based on these models will be presented in Section V.

Table 1 Target Motion Models

\begin{tabular}{|c|c|c|c|}
\hline & Filter Motion Model & Filter State Vector & $\begin{array}{c}\text { State } \\
\text { Dimension }\end{array}$ \\
\hline \hline 1 & White Noise Acceleration & {$[x, y, z, \dot{x}, \dot{y}, \dot{z}]^{T}$} & 6 \\
\hline 2 & Wiener Process Acceleration & {$[x, y, z, \dot{x}, \dot{y}, \dot{z}, \ddot{x}, \ddot{y}, \ddot{z}]^{T}$} & 9 \\
\hline 3 & Ballistic Reentry Vehicle & {$\left[x, y, z, \dot{x}, \dot{y}, \dot{z}, a_{x}, a_{y}, a_{z}\right]^{T}$} & 9 \\
\hline 4 & Spiral & {$\left[x, y, z, \dot{x}, \dot{y}, \dot{z}, Z_{1}, Z_{2}, C_{D m}, \omega\right]^{T}$} & 10 \\
\hline 5 & Generalized Snap & {$\left[x, y, z, \dot{x}, \dot{y}, \dot{z}, a_{x}, a_{y}, a_{z}, \dot{a}_{y}, \dot{a}_{z}, k_{y}, k_{z}, b_{y}, b_{z}\right]^{T}$} & 15 \\
\hline
\end{tabular}

The WNA model is the simplest, while the GS model provides the most detail. In general, more sophisticated motion models are capable expressing a more complex target motion. However, such sophisticated models have a risk of over-parameterization which may lead to lack of observability due to state redundancy, especially when the target motions are relatively simple. Potential filter instability due to the over-parameterization can be suppressed to some extent by introducing a small fictitious damping to the filter system.

\section{Performance Assessment of the Motion Models}

The performance of the motion models outlined in Section IV is investigated through numerical simulations. The target trajectories are generated from the full 6-DOF nonlinear simulation model, and the tracking filters incorporating the proposed motion models estimate the target trajectories based on noise corrupted sensor measurements of the target. The extended Kalman filter (EKF) and unscented Kalman filter (UKF) formulations are used in these assessments.

The four measurement channels that are assumed to be available are range, range rate and the bearing angles in azimuth and elevation. 


\section{A. Reentry Target Specifications}

The physical specifications for the conical reentry vehicle ${ }^{22}$ are used in the present study. Table 2 describes the parameters of this vehicle.

Table 2 Target specifications

\begin{tabular}{||c|c|c|c|c|}
\hline \hline Mass & Ref. Diameter & Ref. Area & $I_{x}$ & $I_{y}=I_{z}$ \\
\hline $673.6(\mathrm{~kg})$ & $0.9144(\mathrm{~m})$ & $0.6567\left(\mathrm{~m}^{2}\right)$ & $42.24\left(\mathrm{~kg}-\mathrm{m}^{2}\right)$ & $197.68\left(\mathrm{~kg}-\mathrm{m}^{2}\right)$ \\
\hline
\end{tabular}

The associated aerodynamic coefficients varying with Mach number are given as a tabular data ${ }^{22}$. The normal and axial force coefficients are obtained from the tabular data based on the current speed and altitude, and then converted into the aerodynamic derivatives shown in Eqn. (3).

\section{B. Tracking Simulations using an EKF}

Tracking simulations were conducted for two simple but distinctively different reentry trajectories. The standard EKF algorithm is used with white Gaussian measurement noise in the present study.

The first is a non-maneuvering ballistic target with symmetric configuration and no initial roll rate. The second trajectory is that of a target with asymmetric configuration (e.g., non-zero pitching moment at zero-angle of attack) and non-zero initial roll rate. The former provides a smooth free-fall trajectory and the latter exhibits complex spiraling motions during the descent. Table 3 describes the initial conditions for the simulations. Fig. 1 and Fig. 2 give the target trajectories and drift angles in the pitch and yaw axes during reentry.

\section{Table 3 Initial conditions}

\begin{tabular}{||c|c|}
\hline Position $(x, y, z)$ & $(-50 \mathrm{~km},-5 \mathrm{~km},-50 \mathrm{~km})$ \\
Speed along $x$-axis & $1500(\mathrm{~m} / \mathrm{s})$ \\
Descent angle & $10(\mathrm{deg})$ \\
Roll rate (Scenario 1) & $0(\mathrm{deg} / \mathrm{s})$ \\
Roll rate (Scenario 2) & $180(\mathrm{deg} / \mathrm{s})$ \\
\hline
\end{tabular}

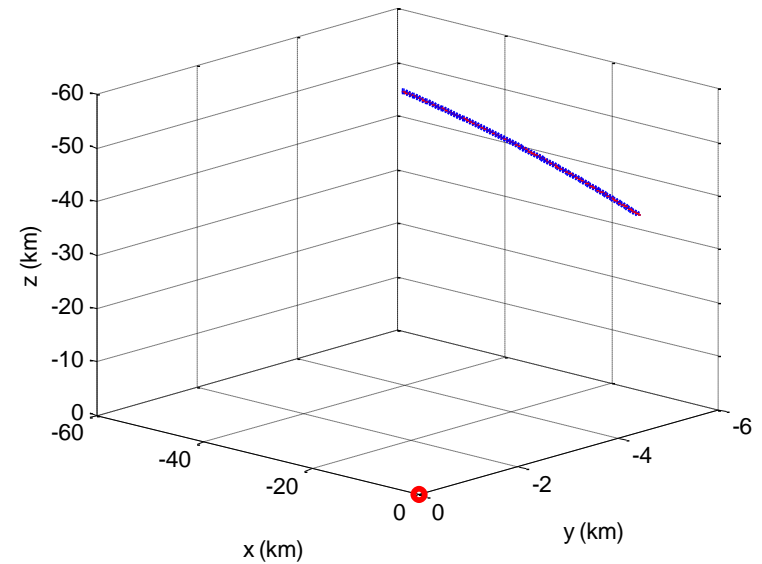

(a) Target trajectory

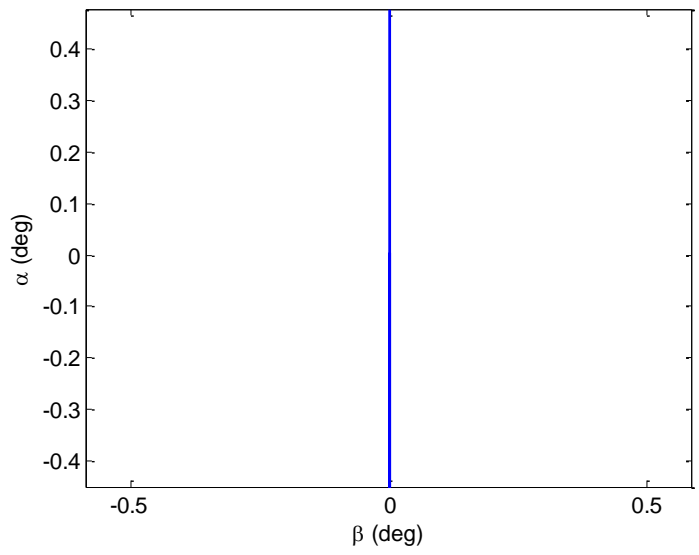

(b) Drift angle plot $(\alpha-\beta)$

Fig. 1 Simulation Scenario 1: Symmetric configuration with no rolling motion

The rolling target with axially asymmetric configuration shows a complex spiral-like behavior as shown in Fig. 2-(b). 


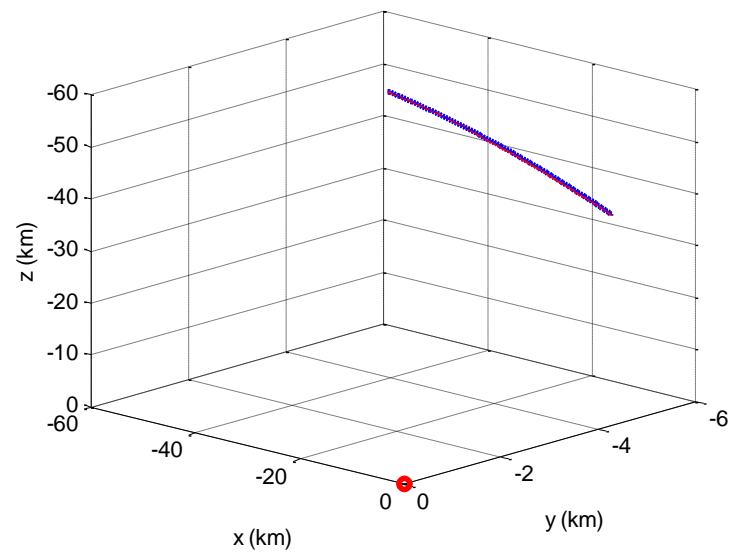

(a) Target trajectory

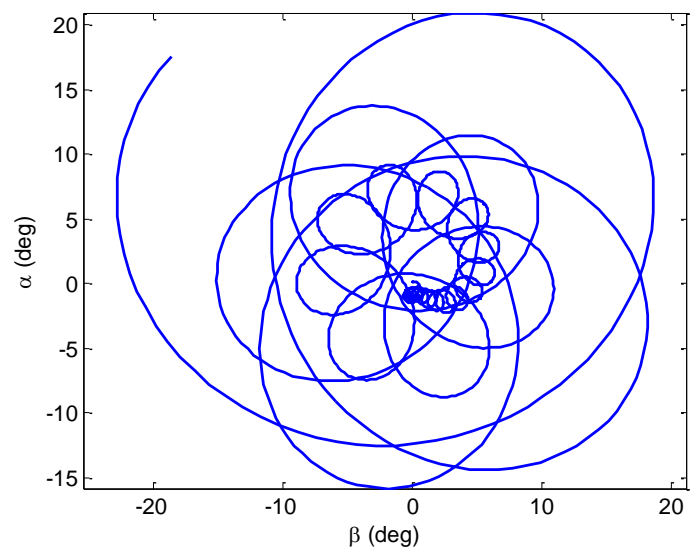

(b) Drift angle plot $(\alpha-\beta)$

Fig. 2 Simulation Scenario 2: Asymmetric configuration with rolling motion

The origin in the trajectory plots represents the observer's position. For the purposes of assessing the performance of motion models, the measurements are assumed to be obtained by a stationary observer.

Two sets of tracking simulations for each motion model have been performed using the EKF formulation. The simulation results are summarized in Table 4.

Table 4 Performance comparison between the motion models

\begin{tabular}{||c|c|c|c||c|c||}
\hline \multirow{2}{*}{ Motion Model } & \multicolumn{2}{|c||}{ Scenario 1 (Free-fall trajectory) } & \multicolumn{2}{c||}{ Scenario 2 (Spiral trajectory) } \\
\cline { 3 - 6 } & & $\begin{array}{c}\text { Position Error } \\
\text { (RMS, m) }\end{array}$ & $\begin{array}{c}\text { Velocity Error } \\
\text { (RMS, } m \text { ) }\end{array}$ & $\begin{array}{c}\text { Position Error } \\
\text { (RMS, } m \text { ) }\end{array}$ & $\begin{array}{c}\text { Velocity Error } \\
\text { (RMS, } \mathrm{m} \text { ) }\end{array}$ \\
\hline \hline 1 & WNA & 10.10 & 24.39 & 10.07 & 24.54 \\
\hline 2 & WPA & 8.75 & 6.83 & 8.72 & 10.11 \\
\hline 3 & BRV & 8.56 & 4.56 & 10.12 & 11.31 \\
\hline 4 & Spiral & 8.06 & 5.89 & 5.19 & 7.51 \\
\hline 5 & Generalized Snap & 8.76 & 3.40 & 7.59 & 19.21 \\
\hline
\end{tabular}

The absolute magnitudes of the estimation errors shown in the above table are not small enough for successful kinetic kill. However, these large errors are mainly due to the large distance between the target and the observer on the ground. Much smaller errors can be obtained if sensor measurements are provided by the seeker of the kill vehicle at relatively close range to the target.

Though the tracking performance does not vary significantly between the motion models, slightly improved performance is observed as the motion model gets more sophisticated. Relatively large errors in velocity estimates for the second scenario are seen, which can be attributed to the inherent motion complexity of a spiraling target.

\section{Monte-Carlo Simulations: Combined Trajectories of Spiraling-Random Telegraph-Weaving}

Since target state estimation involves random noise components, conclusions must be made based on MonteCarlo (MC) simulations with a sufficient number of test runs. Here, a number of simulations are performed, and their statistical results are compared. For recursive filtering, two widely-used nonlinear estimators, the Extended Kalman Filter (EKF) and the unscented Kalaman filter (UKF) algorithms are considered. As stated at the beginning of this section, a Gaussian sensor noise model was used in the present MC simulations. 
A more complex nominal reentry trajectory is designed for the MC simulations, which includes spiraling, random telegraph, and weaving motions. The target reentry trajectory is a $3 \mathrm{D}$ space and its linear/angular acceleration time histories are shown in Fig. 3 and Fig. 4.

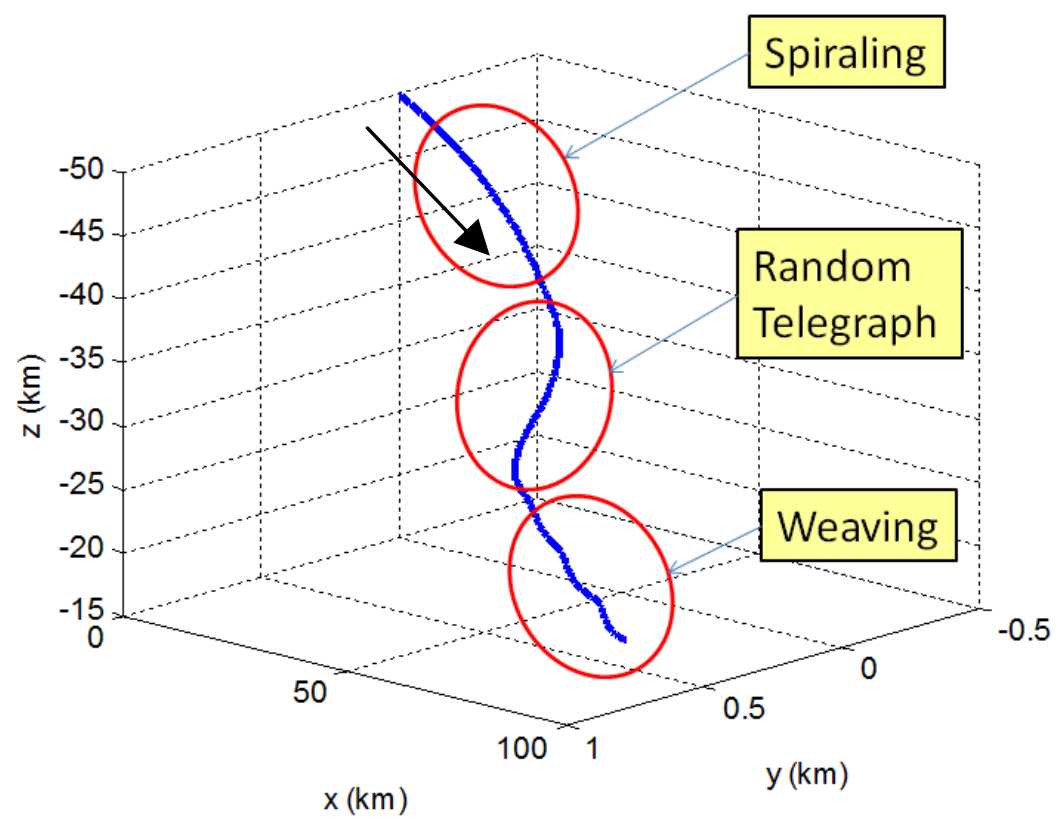

Fig. 3 Combined mode target trajectory
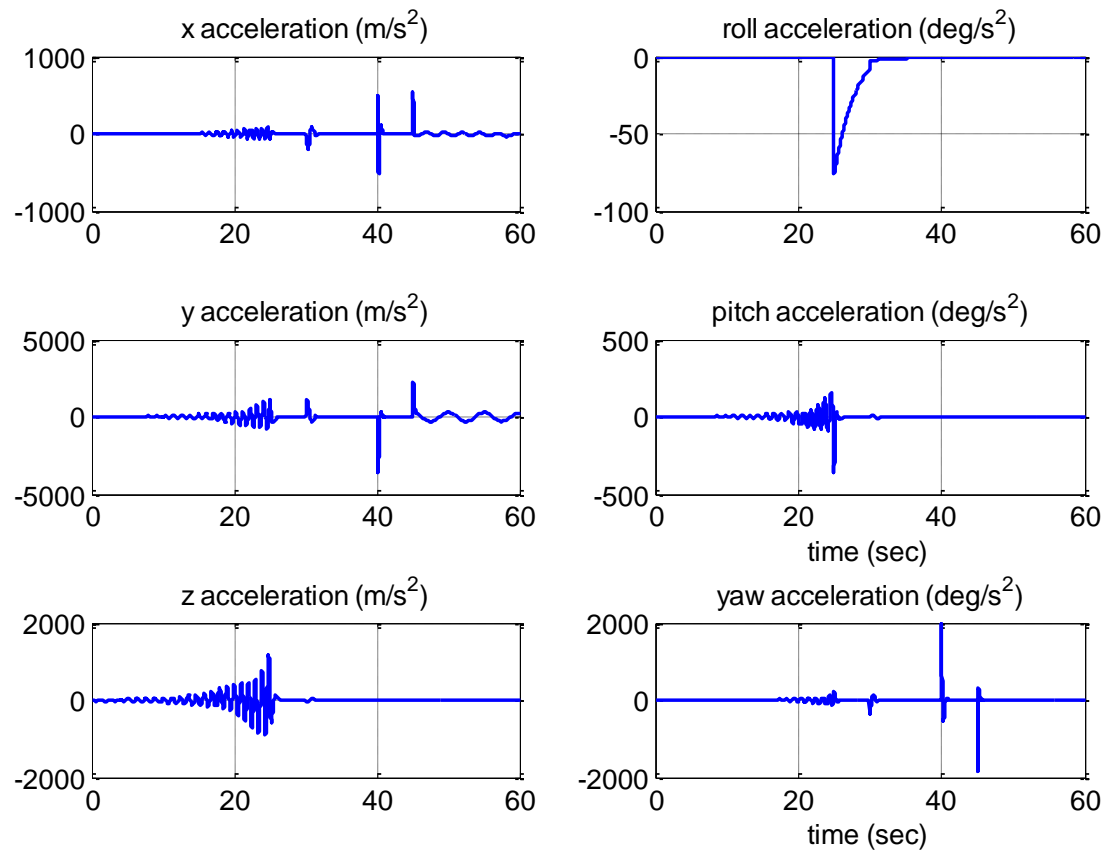

Fig. 4 Linear/angular acceleration time histories of the target trajectory

A total of 1000 simulation runs for the five different motion models have been made, which are 100 runs per each motion mode using an EKF and another 100 using an UKF. The quantitative results from these simulations are summarized in Table 5. 
Table 5 Monte-Carlo simulations with the combined motion trajectory

\begin{tabular}{|c|c|c|c|c|c||}
\hline \multirow{2}{*}{} & \multirow{2}{*}{ Motion Model } & \multicolumn{2}{|c|}{ Position RMS Errors (m) } & \multicolumn{2}{c|}{ RMS Errors (normalized) } \\
\cline { 3 - 6 } & & EKF & UKF & EKF & UKF \\
\hline 1 & WNA & 10.0430 & 10.0429 & 1.0000 & 1.0000 \\
\hline 2 & WPA & 9.7296 & 9.7296 & 0.9688 & 0.9688 \\
\hline 3 & BRV & 9.6138 & 9.2949 & 0.9573 & 0.9255 \\
\hline 4 & Spiral & 9.0628 & 9.0598 & 0.9024 & 0.9021 \\
\hline 5 & Generalized Snap & 9.2976 & 9.0143 & 0.9258 & 0.8976 \\
\hline
\end{tabular}

Table 5 shows the RMS errors in position estimate. The main purpose of this simulation is to compare the performance between the motion models. Thus, for a more clear comparison, the errors are normalized using the baseline RMS error by the EKF with the simplest WNA motion model in Table 5, and graphically illustrated in Fig. 5.

According to Fig. 5, the RMS errors generally decrease as the complexity of the proposed motion models increases. One exception to this trend is that the generalized snap (GS) model performs more poorly than the spiral model when used with the EKF. However, this anomaly is believed to be due to the limitation of the EKF, not of the motion model itself. In fact, the UKF, which is known to be more capable than the EKF in handling nonlinear systems, has always performed better then the EKF in the above simulations, and the GS model with the UKF shows a slightly better performance than the spiral model with the UKF as well.

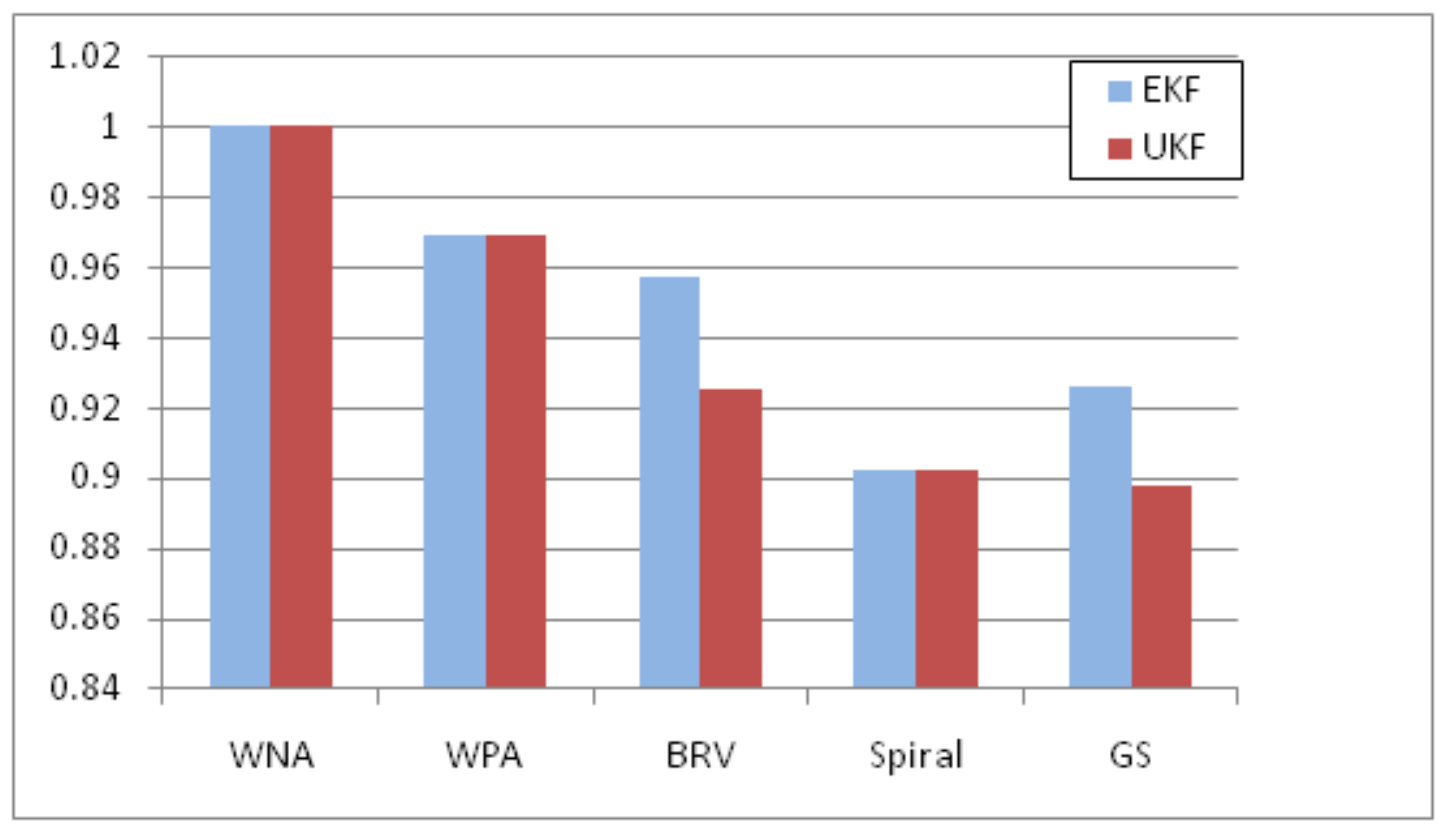

Fig. 5 Normalized Position RMS error comparison between the motion models

\section{Filtering/Prediction Simulation}

In order to examine the quality of predications using the proposed motion model, a filtering simulation is performed for a fixed amount of time with a normal measurement update step (i.e., filtering phase), followed by a prediction simulation for the next two seconds during which measurements are not provided to the filter (i.e., 
prediction phase). An oscillatory trajectory was created and used to compare the predictions between the BRV model and the proposed GS model. These are given in Fig. 6.

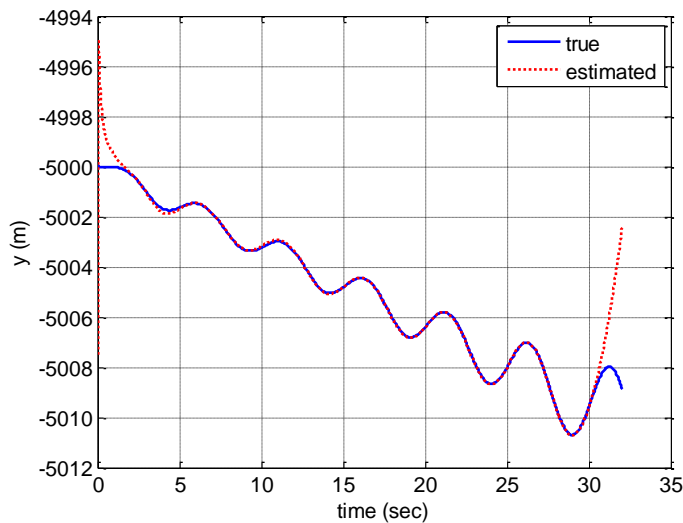

(a) Ballistic reentry vehicle (BRV) model

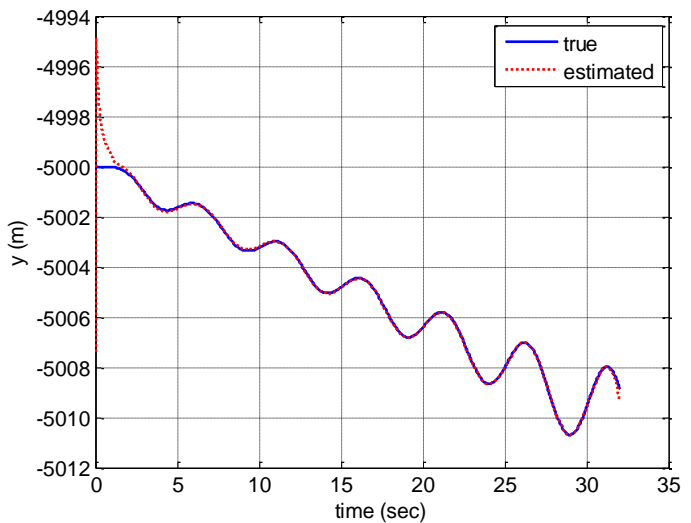

(b) Generalized snap model

Fig. 6 Filtering of weaving motion followed by trajectory prediction

For both plots in Fig. 6, the prediction starts at $\mathrm{t}=30$ (sec) and lasts for 2 seconds. The figures clearly illustrate that the generalized snap model performs better than the BRV model. The state estimate during the prediction phase is solely determined by the filter motion model with the internal system parameters which have been tuned using sensor measurements during the filtering phase. The generalized snap motion model has a mathematical structure that can capture the oscillatory maneuver patterns, while the BRV model does not. The BRV model provides a prediction expecting no further accelerations, and, basically, all the linear wide-band motion models work in a similar fashion.

\section{Conclusions}

The main objective of this research is the development of a target state estimation algorithm for tracking a ballistic target. Primary research emphasis was placed upon developing a target motion model that is capable of handling a wide variety of maneuvers such as weaving, random telegraph and spiraling.

In order to fully exploit the information on the typical dynamic behavior of the ballistic target, the mathematical modeling and dynamic analysis of the aerodynamic model of the ballistic target were carried out. Based on this, a new motion model, called the generalized snap model was derived. The performance of the new model was compared with other motion models through extensive numerical simulations, and some advantages and potentials were found.

The proposed motion model better approximates the actual phenomena and generally outperforms the linear wide-band models. This model has several parameters to describe the internal dynamics of the target, which leads to a state-parameter estimation problem involving high nonlinearity. The EKF algorithm was found to be ineffective in handling such high nonlinearity, and the UKF, which is known to have better nonlinear approximation ability, showed better performance. This observation leads to a conjecture that a better nonlinear filtering technique such as the particle filter may be capable of fully exploiting the benefit of using sophisticated motion models including the proposed GS model. Future research is highly recommended to further investigate this issue.

\section{Acknowledgments}

This research was supported under MDA SBIR Contract No. HQ0147-09-C-7111 with Dr. Demetrios Serakos of Naval Surface Warfare Center as the Technical Monitor. We thank Dr. Serakos for his interest and support of this research.

\section{References}

${ }^{1}$ Bryson, A. B. and Ho, Y. C., Applied Optimal Control, Hemisphere, 1975, pp. 422-428.

2 Terejanu, G., Singla, P., Singh T. and Scott, P. D., "Uncertainty Propagation for Nonlinear Dynamical Systems using Gaussian Mixture Models," AIAA Guidance, Navigation and Control Conference, Honolulu, HI, 2008.

${ }^{3}$ Gelb, A., Applied Optimal Estimation, The M.I.T. Press, 1974.

${ }^{4}$ Maybeck, P. S., Stochastic Models, Estimation, and Control, Academic Press, 1979. 
${ }^{5}$ Vaddi, S. S., Menon, P. K. and Ohlmeyer E. J., "Target State Estimation for Integrated Guidance-Control of Missiles," AIAA Guidnace, Navigation, and Control Conference, Hilton Head, SC, 2007.

${ }^{6}$ Bar-Shalom, Y., Li X. R. and Kirubarajan, T., Estimation with Applications to Tracking and Navigation, John Wiley \& Sons, 2001.

${ }^{7}$ Li, X. R. and Jilkov, V. P., "Survey of Maneuvering Target Tracking - Part I: Dynamic Models," IEEE Trans. Aerospace and Electronic Systems, Vol. 39, No. 4, pp. 1333-1364, 2003.

${ }^{8} \mathrm{Li}, \mathrm{X}$. R. and Jilkov, V. P., "Survey of Maneuvering Target Tracking - Part II: Ballistic Target Models," 2001 SPIE Conference on Signal and Data Processing of Small Targets, San Diego, CA, USA, 2001.

${ }^{9}$ Rago, C. and Mehra, R. K., "Robust Adaptive Target State Estimation for Missile Guidance Using the Interacting Multiple Model Kalman Filter," IEEE Position Location and Navigation Symposium (PLAN 2000), San Diego, CA, 2000.

${ }^{10}$ Farina, A., Immediata, S., and Timmoneri, L., "Impact of Ballistic Target Model Uncertainty on IMM-UKF and IMM-EKF tracking accuracies," 2006 European Association for Signal Processing (EURASIP), Florence, Italy, 2006.

${ }^{11} \mathrm{Li}$, X. R. and Jilkov, V. P., "Survey of Maneuvering Target Tracking - Part V: Multiple Model Methods," IEEE Trans. Aerospace and Electronic Systems, Vol. 41, No. 4, pp. 1255-1321, 2005.

${ }_{12}$ Astrom, K. J. and Wittenmark, B., Adaptive Control, Addision-Wesley, 1995.

${ }^{13}$ Menon, P. K. et al., Robust Target State Estimation Using Adaptive Maneuver Models, Report No. OSI-NANY-98010, Optimal Synthesis Inc., 1999.

${ }^{14}$ Kim, J., Vaddi, S. S., Menon, P. K. and Ohlmeyer E. J., "Comparison between Three Spiraling Ballistic Missile State Estimators," Proc. AIAA Guidance, Navigation and Control Conference, Honolulu, HI, 2008.

${ }^{15}$ Chadwick, W. R. and Zarchan, P., "Interception of Spiraling Ballistic Missiles," American Control Conference, Seattle, WA, 1995.

${ }^{16}$ Chadwick, W. R., "Flight Dynamics of a Bomb with Cruciform Tail," AIAA Journal of Spacecraft and Rockets, Vol. 4, No. 6, pp 768-773, 1967.

${ }^{17}$ Platus, D. H., "Ballistic Re-entry Vehicle Flight Dynamics," AIAA Journal of Guidance, Control, and Dynamics, Vol. 5, No. 1, pp 4-16, 1982.

${ }^{18}$ Zarchan, P., "Tracking and Intercepting Spiraling Ballistic Missiles," IEEE Position Location and Navigation Symposium, 2000.

${ }^{19}$ Greenwood, D. T., Principles of Dynamics, 2nd Ed., Prentice Hall, 1988.

${ }^{20}$ Blakelock, J. H., Automatic Control of Aircraft and Missiles, 2nd Ed., John Wiley \& Sons, 1991.

${ }^{21}$ Siouris, G. M., Missile Guidance and Control Systems, Springer-Verlag, 2004.

${ }^{22}$ Menon, P. K. and Sweriduk G. D., Integrated Guidance and Control of Moving Mass Actuated Kinetic Warheads, Final Report prepared under Navy Phase I SBIR Contract No. N00178-01-C-1020, Optimal Synthesis Inc., 2001 